\title{
The array for investigations of EAS neutron component
}

\author{
D.M. Gromushkin ${ }^{1, a}$, N.V. Ampilogov ${ }^{1}$, N.S. Barbashina ${ }^{1}$, F.A. Bogdanov ${ }^{1}$, R.P. Kokoulin ${ }^{1}$, V.V. Ovchinnikov ${ }^{1}$, \\ A.A. Petrukhin ${ }^{1}$, I.A. Shulzhenko ${ }^{1}$, Yu.V. Stenkin ${ }^{1,2}$, I.I. Yashin ${ }^{1}$, and K.O. Yurin ${ }^{1}$

\footnotetext{
${ }^{1}$ National Research Nuclear University MEPhI (Moscow Engineering Physics Institute), 115409 Moscow, Russia
} \\ ${ }^{2}$ Institute for Nuclear Research of RAS, Moscow 117312, Russia
}

\begin{abstract}
The neutron component of EAS has not been practically studied for many years. Creation of the neutron detector arrays PRISMA-32 and PRISMA-YBJ demonstrated the effectiveness of such investigations. It is important to underline that recording the EAS neutron component is carried out over the whole array area. Development of a new larger scale neutron detector array (URAN) in MEPhI will continue these studies. During the construction of the URAN array, a new design of en-detectors based on the scintillator with natural boron and new electronics was developed. The use of new electronics with a switchable sampling rate allows one to reach EAS fast timing accuracy up to $10 \mathrm{~ns}$. The en-detectors of the URAN are located on the roofs of the buildings. The sensitive area of each detector is $0.36 \mathrm{~m}^{2}$, while the array's starting area is $\sim 10^{3} \mathrm{~m}^{2}$. Total number of the initial array detectors is 72. They are subdivided into 6 independent clusters each of 12 en-detectors, connected with the central data acquisition station.
\end{abstract}

\section{Introduction}

The neutron component of EAS is produced in interactions of the hadronic component with atmospheric nuclei or substance close to the detector. In its turn, the hadron component carries important information about the primary particle energy. Therefore, studying it is of a great interest. Investigations of the hadronic component are mainly performed using hadron calorimeters, but they are quite expensive and have a limited area. In 2001, a new method for the registration of EAS hadronic component using thermal neutrons was proposed [1]. The first results on the EAS neutrons were obtained at the PRISMA32 setup [2] (MEPhI, Russia). The obtained data have shown the possibilities of studying the EAS neutrons using the proposed method [3-5]. The main feature of this method is the usage of en-detectors which register EAS electromagnetic and neutron components simultaneously.

In 2014-2015 in MEPhI within the frameworks of the Program for support of the Russian Unique Scientific Facilities the first stage of a new URAN (Upstairs Registration of Atmospheric Neutrons) array [6] was created. It is aimed at the registration of thermal neutrons produced by the EAS hadronic component over the whole area of the array. The URAN detectors are located on the roofs of MEPhI laboratory buildings. Such location allows to decrease the influence of the building floor structure.

\section{The URAN array}

The first stage of the URAN array includes 72 en-detectors. The detectors are located on the roofs of two laboratory buildings and are combined into cluster structures. On each roof, three clusters (36 en-detectors) are deployed. The total area of the array is about $10^{3} \mathrm{~m}^{2}$. Figure 1 shows the layout of the URAN array.

\subsection{Detectors}

The en-detector is a basic element of the URAN array. The construction of the en-detector is shown in Fig. 2. A scintillator with an area of $0.36 \mathrm{~m}^{2}$ is mounted at the bottom of a 200-liter plastic tank [7]. In order to enhance the light collection, a cone with a diffusely reflecting surface is used. In the cone vertex an FEU-200 PMT (Novosibirsk, Russia) is installed. The PMT is attached to the tank lid with a diameter of $350 \mathrm{~mm}$. For protection against environmental influences, detectors are installed inside the external housings made of $0.7 \mathrm{~mm}$ galvanized steel.

For the registration of thermal neutrons produced by the EAS high-energy hadrons, an inorganic scintillator based on $\mathrm{ZnS}$ with the addition of $\mathrm{B}_{2} \mathrm{O}_{3}$ is used. The content of ${ }^{10} \mathrm{~B}$ in $\mathrm{B}_{2} \mathrm{O}_{3}$ is $18 \%$. This scintillator has the following features:

- high light yield at the registration of heavy particles produced during neutron capture;

- sufficiently high effectiveness of thermal neutron registration due to the large cross-section of thermal neutron capture by ${ }^{10} \mathrm{~B}$ nuclei;

- fast response (40 ns);

- low sensitivity to single charged particles.

Registration of thermal neutrons in the scintillator $\mathrm{ZnS}(\mathrm{Ag})+\mathrm{B}_{2} \mathrm{O}_{3}$ occurs due to the following reactions:

$$
\begin{aligned}
{ }^{10} \mathrm{~B}+\mathrm{n} \rightarrow & { }^{7} \mathrm{Li}+\alpha+2.792 \mathrm{MeV} \\
{ }^{10} \mathrm{~B}+\mathrm{n} \rightarrow & { }^{7} \mathrm{Li}^{*}+\alpha+2.31 \mathrm{MeV} \\
& \downarrow \\
& { }^{7} \mathrm{Li}^{*} \rightarrow{ }^{7} \mathrm{Li}+\gamma+482 \mathrm{keV} .
\end{aligned}
$$

a e-mail: DMGromushkin@mephi.ru

(c) The Authors, published by EDP Sciences. This is an Open Access article distributed under the terms of the Creative Commons Attribution License 4.0 (http://creativecommons.org/licenses/by/4.0/). 


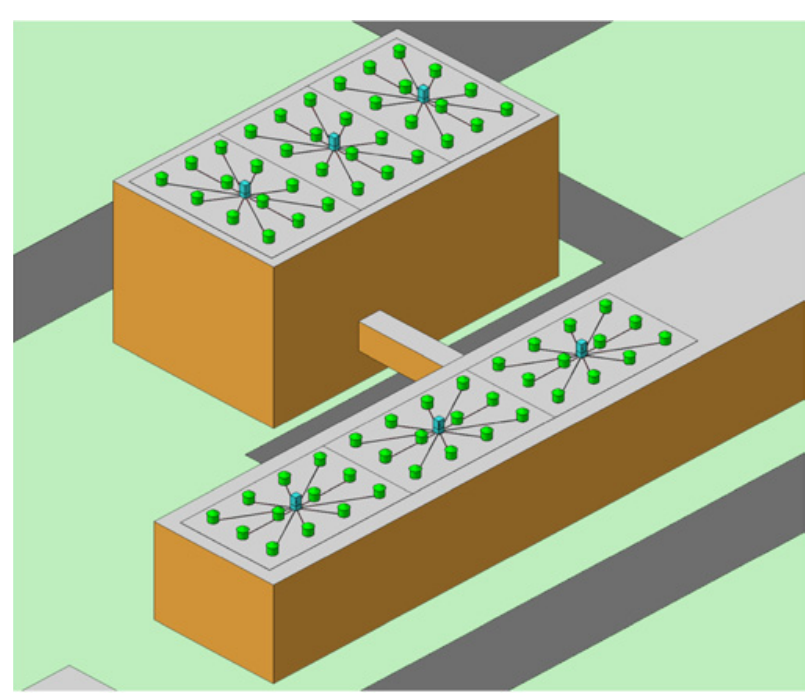

Figure 1. The layout of the URAN array.

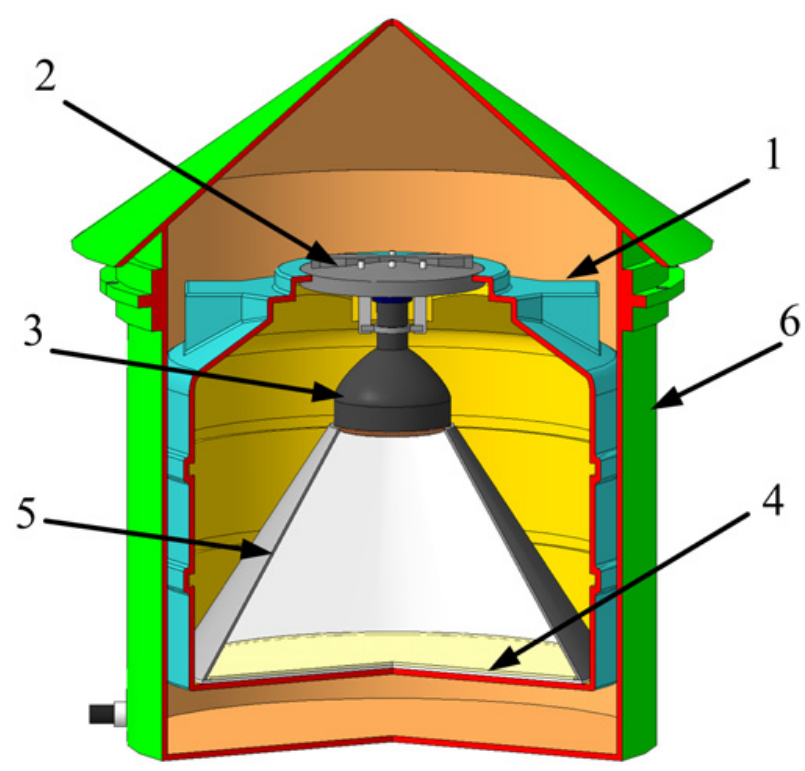

Figure 2. The en-detector design; 1 - light protecting housing; 2 - tank lid; 3 - FEU-200 PMT; 4 - scintillator; 5 - light reflecting cone, 6 - outer metal housing.

The formed nuclei cause luminosity of $\mathrm{ZnS}(\mathrm{Ag})$ in the visible wavelength range [8]. Due to the use of $\mathrm{ZnS}(\mathrm{Ag})$, the detectors also allow to register the EAS electron component at the group passage of charged particles.

Before the array deployment, all detectors were tested and adjusted. The response of en-detectors to the neutron registration was studied using the test facility allowing to separate neutron and charged particle signals according to the pulse shape. The pulse from the neutron capture products has a longer rise time (several $\mu \mathrm{s}$ ) than a pulse from charged particles or PMT noise due to a more intensive luminosity of the scintillator slow components. In order to increase statistics and to decrease the influence of meteorological factors, a neutron source ${ }^{252} \mathrm{Cf}$ (with neutron flux of $\sim 1000 \mathrm{~s}^{-1}$ ) is installed under the detector. To obtain thermal-energy neutrons, the source is placed in a cube made of a hydrogen-containing substance (polyethylene with a width of $100 \mathrm{~mm}$ ).

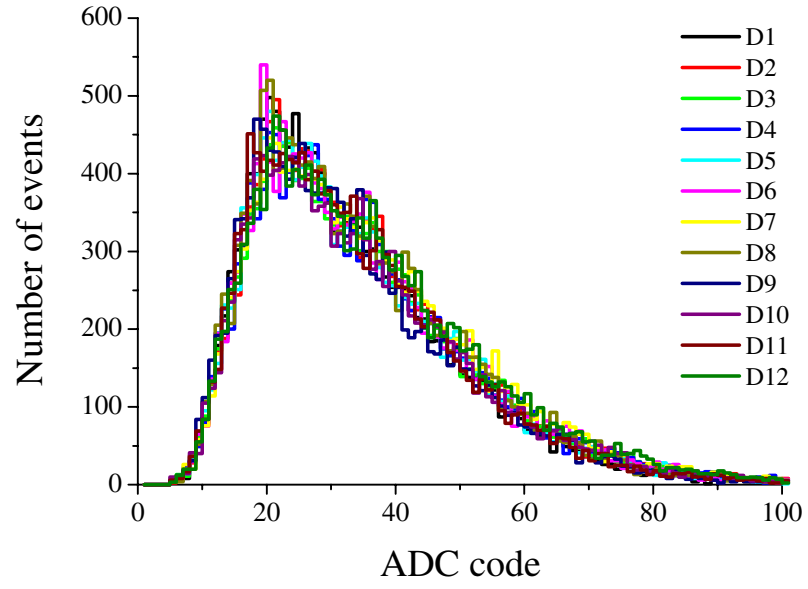

Figure 3. Neutron signal spectra for 12 detectors.

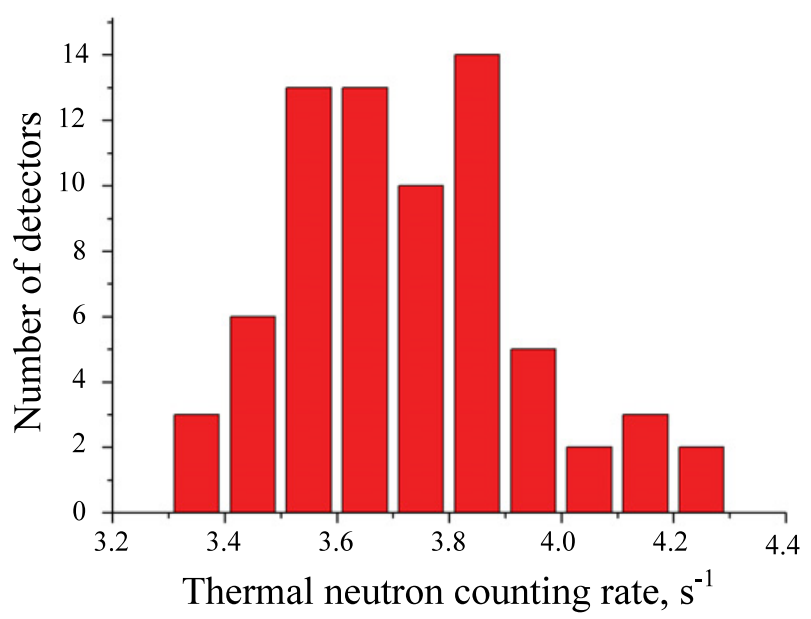

Figure 4. Distribution of 72 detectors in the thermal neutron counting rates.

En-detector testing included 1-hour measurements of the thermal neutron amplitude spectra. An example of the measured spectra for 12 detectors is shown in Fig. 3. For better separation, signals are integrated with an integration constant of $20 \mu \mathrm{s}$. The obtained amplitude spectra are similar. The maxima are located at about $25^{\text {th }}$ code of ADC.

Figure 4 shows the distribution of 72 en-detectors in the thermal neutron counting rates. The average thermal neutron counting rate is 3.7 neutrons/s, maximal counting rate deviation for all tested en-detectors does not exceed $14 \%$.

\subsection{Electronics}

Electronics for signal digitizing is installed in the Local Post (LP) of primary data processing which, like the detectors, is located on the building roof. Cluster electronics includes two 12-channel blocks of amplitude analysis designed according to the $6 \mathrm{U}$ Euromechanics standard. One block performs digitizing of analog signals from the $12^{\text {th }}$ dynodes of the cluster PMTs, the second block digitizes analog signals from the $7^{\text {th }}$ dynodes. Usage of a two-dynode readout system allows to increase the dynamic range for measuring the energy deposit of the EAS electromagnetic component. The structural 


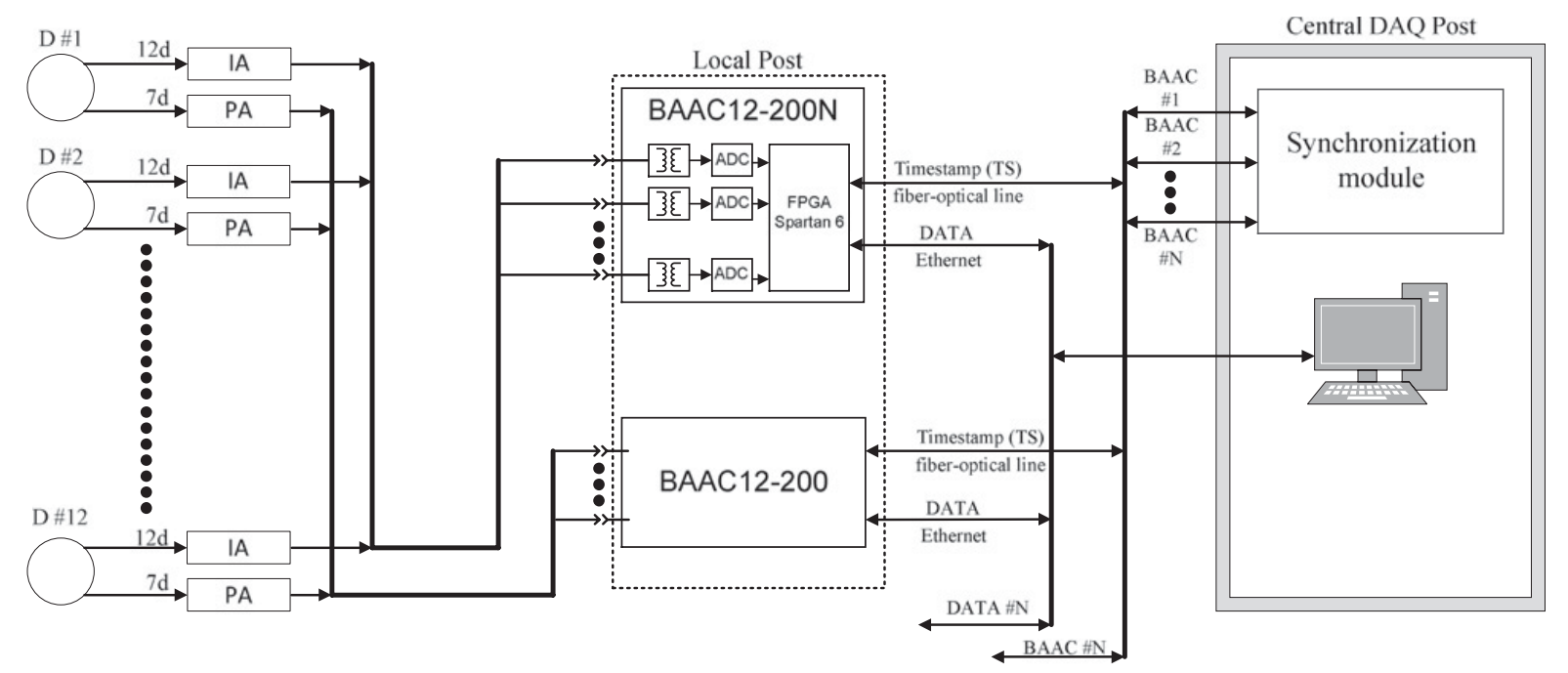

Figure 5. Structural scheme of the URAN array registering system.

scheme of the URAN array electronics is shown in Fig. 5. Signals from the detectors are fed to the inputs of the block of amplitude analysis via $25 \mathrm{~m}$ coaxial cables. Beside this, signals from the $12^{\text {th }}$ dynodes are fed through the integrator-amplifier with an integration time of $1 \mu \mathrm{s}$, and signals from the $7^{\text {th }}$ dynodes - through the preamplifier. Integrator-amplifier (IA) and preamplifier (PA) are installed inside the external housing of the detector. Both blocks have the same design, but differ in the software configuration. Block ADCs digitize analog signals from the en-detectors with a sampling frequency of $200 \mathrm{MHz}$ in the amplitude range from $-3.5 \mathrm{~V}$ to $+3.5 \mathrm{~V}$.

The main block digitizing signals from the $12^{\text {th }}$ dynodes constantly records data into the 1024-count circular buffer. If the triggering conditions are fulfilled, the sampling frequency changes from $200 \mathrm{MHz}$ to $1 \mathrm{MHz}$ and 20 thousand counts are recorded. Due to such a digitizing scheme, the first 1024 points are recorded with a step of $5 \mathrm{~ns}$. This allows to obtain information about the energy deposit of the electromagnetic component. The following 20000 points digitized with a step of $1 \mu \mathrm{s}$ provide information about thermal neutrons accompanying the shower. The second block has no sampling frequency variation and only records 1024 points with a step of $5 \mathrm{~ns}$ (information on the energy deposit of the electron component comes from the $7^{\text {th }}$ dynode of the PMT).

Blocks operate independently from each other. The data of separate clusters are associated according to the time of the cluster event registration. The common time for all blocks is set by the synchronization module which broadcasts the unified clock signal with a frequency of $100 \mathrm{MHz}$ via a fiber-optical communication line and ensures simultaneous starting of internal clocks of all blocks. Time synchronization accuracy is $10 \mathrm{~ns}$.

Such a registering system allows to receive and store data in the form of oscillograms of all detectors for each event (data are stored in binary files).

The experimental data processing is performed by special software in the off-line mode. This software allows to obtain information about registered EAS: the energy deposit of electromagnetic component and the number of neutrons detected during $20 \mathrm{~ms}$ after the EAS front arrival. The oscillogram of the registered thermal neutron is shown in Fig. 6.

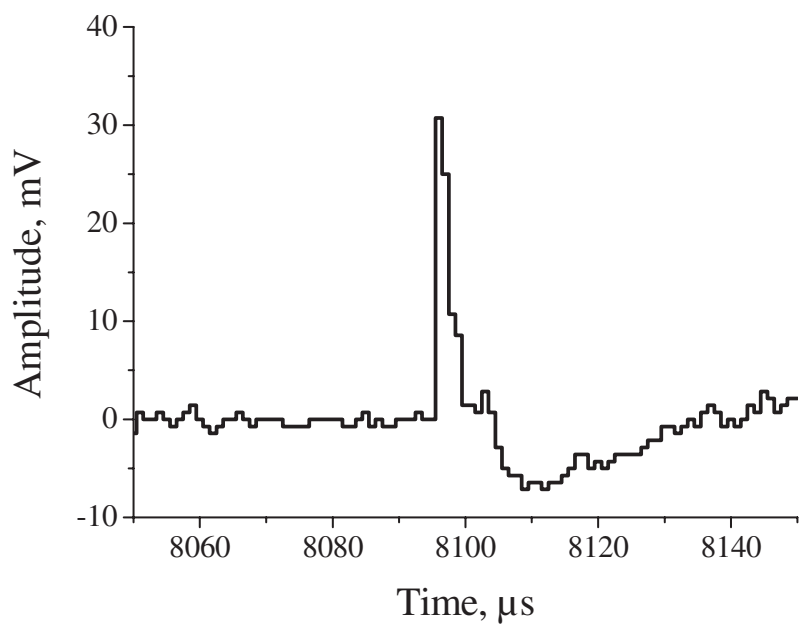

Figure 6. Example of the oscillogram of the neutron pulse integrated over $1 \mu \mathrm{s}$.

\section{Test run}

For studying the operation of en-detectors, a test run with the first three clusters of the URAN array was started. During the test run, all detectors and electronics blocks were examined, the registration thresholds for EAS events were adjusted. At the next step, a week-long exposure was started. The cluster triggering condition was a coincidence of not less than 2 en-detectors with signal amplitudes of at least $12 \mathrm{mV}$.

During the test run, the first EAS events were registered. Figure 7 shows an example of the event registered by 3 clusters of the URAN array. The responses of en-detectors to the EAS electromagnetic component $A_{e}$ (in ADC codes) and the numbers of neutrons registered during $20 \mathrm{~ms}$ after the shower front arrival are indicated. In this event, 36 detectors registered 24 neutrons. Using the values of responses to the electromagnetic component, the EAS axis position can be estimated (marked by a cross in Fig. 7).

The events with high energy deposit of the EAS electromagnetic component have been associated with events registered by other setups of the NEVOD complex (PRISMA-32 [2], NEVOD-EAS [9,10] and 


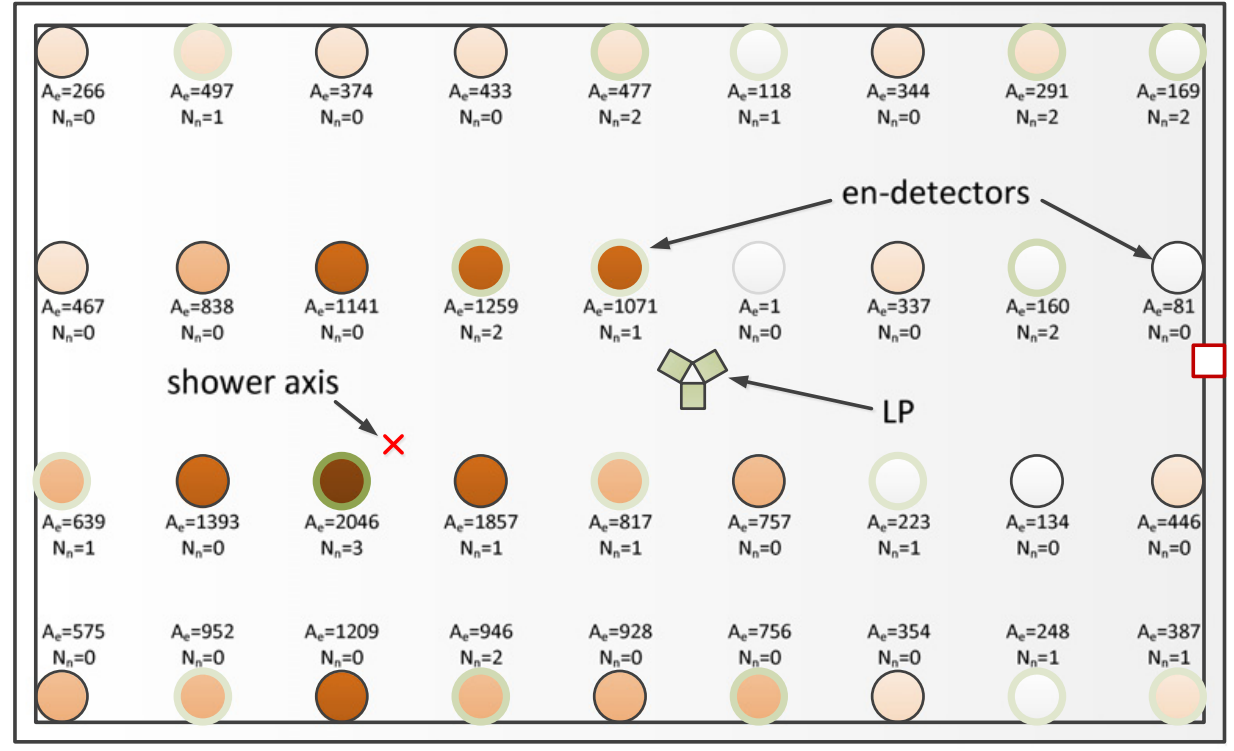

Figure 7. Example of the EAS registration with three clusters of the URAN array.

NEVOD-DECOR-CTS [11]). It has been revealed that the topologies of the events are in good agreement with each other. In the near future, a system of global synchronization with the NEVOD-EAS array will be started. It will allow to obtain quantitative estimations of the EAS registration.

\section{Conclusion}

The first stage of the setup for registration of EAS neutrons has been created. Test runs have been started. The first EAS events registered during test runs are in good agreement with the data of other installations. The URAN array is an inseparable part of already existing detectors of the Experimental Complex NEVOD and installations which are now under construction. The combination of setups using different methods of EAS registration into a single experimental structure will allow to carry out multicomponent studies of extensive air showers.

This work was performed at the Unique Scientific Facility "Experimental complex NEVOD" with the support of the Ministry of Education and Science of the Russian Federation (grant RFMEFI59114X0002, MEPhI Academic Excellence
Project 02.a03.21.0005 of 27.08.2013) and the Russian Foundation for Basic Research (grant 16-29-13028-ofi-m).

\section{References}

[1] Yu.V. Stenkin and J.F. Valdes-Galicia, Modern Phys. Letters A 17, 1745 (2002)

[2] D.M. Gromushkin et al., J. Instrumentation 9, C08028 (2014)

[3] D.M. Gromushkin et al., Phys. Atomic Nuclei 78, 349 (2015)

[4] D.M. Gromushkin et al., Astrophys. Space Sci. Trans. 7, 115 (2011)

[5] D.M. Gromushkin et al., J. Phys.: Conf. Ser. 409, 012044 (2013)

[6] D.M. Gromushkin, et al., J. Phys.: Conf. Ser. 675, 032043 (2016)

[7] M.B. Amelchakov et al., PoS (ICRC2015) 651 (2015); http: pos.sissa.it

[8] W.D. Allen, Neutron detection. Harwell (1960)

[9] I.A. Shulzhenko et al., Bull. Russ. Acad. Sci. Phys. 79, 389 (2015)

[10] I.I. Yashin et al., J. Phys.: Conf. Ser. 632, 012029 (2015)

[11] V.V. Kindin et al., Physics Procedia 74, 435 (2015) 